Revue d'histoire de l'Amérique française

REVUE D.HISTOIRE DE L'AMÉRIQUE FRANÇAISE

\title{
JOLOIS, Jean-Jacques, J.-F. Perrault, 1753-1844 et les origines de l'enseignement laïque au Bas-Canada. Les Presses de l’Université de Montréal, 1969. 268 p. \$5.25.
}

\section{Louis-Philippe Audet}

Volume 23, numéro 4, mars 1970

URI : https://id.erudit.org/iderudit/302942ar

DOI : https://doi.org/10.7202/302942ar

Aller au sommaire du numéro

Éditeur(s)

Institut d'histoire de l'Amérique française

ISSN

0035-2357 (imprimé)

1492-1383 (numérique)

Découvrir la revue

Citer ce compte rendu

Audet, L.-P. (1970). Compte rendu de [JOLOIS, Jean-Jacques, J.-F. Perrault, 1753-1844 et les origines de l'enseignement lä̈que au Bas-Canada. Les Presses de l'Université de Montréal, 1969. 268 p. \$5.25.] Revue d'histoire de l'Amérique française, 23(4), 618-625. https://doi.org/10.7202/302942ar d'utilisation que vous pouvez consulter en ligne.

https://apropos.erudit.org/fr/usagers/politique-dutilisation/ 
JoLoIs, Jean-Jacques, J.-F. Perrault, 1753-1844 et les origines de l'enseignement laïque au Bas-Canada. Les Presses de l'Université de Montréal, 1969. 268 pp. \$5.25.

Il faut reconnaître à $\mathrm{M}$. Jean-Jacques Jolois beaucoup de courage et de ténacité, voire même un peu de naïveté dans les efforts qu'il a déployés pour nous convaincre que Joseph-François Perrault fut "l'unique pédagogue du Canada français" (sic) au XIX ${ }^{e}$ siècle! Rien que cela! Depuis que Philippe-Baby Casgrain, dans un ouvrage publié en 1898 sous le titre de La vie de Joseph-François Perrault, surnommé le père de l'éducation 
du peuple canadien, a raconté, assez incomplètement d'ailleurs, la vie mouvementee et pittoresque du protonotaire, tous les historiens de notre organisation scolaire se sont empressés de rendre hommage à ce fonctionnaire qui se découvre, à 70 ans près, une vocation de pédagogue et de philosophe de l'éducation. L'ouvrage de M. Jolois aura le mérite d'avoir apporté un peu plus d'ordre dans le récit des principaux événements de cette longue existence de quatre-vingt-onze ans! Il nous raconte sa jeunesse mouvementée, ses aventures politiques, son travail à la Cour du Banc du Roi; pour résumer cette période, il précise même que Perrault fut successivement aventurier, trafiquant, commerçant, chômeur, politicien, tribun, agitateur politique, capitaine de milice et fonctionnaire prévaricateur (sic), étant accusé d'avoir, entre 1797 et 1802, chargé une double commission (p. 98). C'est cet individu qui, à l'âge de 68 ans (en 1821), va entreprendre la fondation de sociétés d'éducation et d'écoles pour garçons et pour filles, voire d'une école d'agriculture, tout en vaquant (?) à ses devoirs essentiels de protonotaire et de greffier de la Cour du Banc du Roi pour le district de Québec. La fonction publique offrait donc, même à cette époque, des loisirs féconds !

Les chapitres dans lesquels l'auteur nous décrit le rôle de Perrault à la Société d'éducation de Québec (1821 à 1825), à la Société d'école britannique et canadienne (1825 à 1828), ses efforts pour la fondation d'une école de garçons et d'une école de filles à la Haute-ville de Québec (1829 à 1837), son projet d'établir une école d'agriculture (1832 et 1833) et enfin ses offres de service pour la direction des écoles normales en 1836 (il n'avait alors que 83 ans !) constituent une mise en ordre précieuse de renseignements épars que $M$. Jolois a dû compulser, sans doute, dans les documents officiels, notamment dans les Journaux de la Chambre d'Assemblée du Bas-Canada et dans les journaux du temps, particulièrement dans La Gazette de Québec. Enfin, l'étude des méthodes d'enseignement préconisées par Perrault et l'organisation matérielle de ses classes fournissent à l'auteur l'occasion de présenter une comparaison intéressante entre les méthodes québecoises et celles préconisées par Joseph Lancaster, le promoteur de l'enseignement mutuel ou par moniteurs.

Une bibliographie d'une quinzaine de pages offre aux chercheurs une liste précieuse d'ouvrages à consulter, non seulement sur Perrault, mais encore sur l'époque tourmentée durant laquelle il a vécu. Une mention particulière doit être faite de la liste complète des œuvres mêmes du protonotaire (pp. 18-20).

Cet ouvrage, admirablement présenté par les Presses de l'Université de Montréal, exige cependant que nous fassions ici quelques réserves importantes : en premier lieu, l'auteur, qui ne réside au Canada que depuis 1958, présente parfois une interprétation des faits qui n'est pas conforme à l'Histoire; en second lieu il manifeste, tout au long de l'ouvrage, une aggressivité persistante contre le clergé et l'Eglise catholiques qu'il rend responsables de l'analphabétisme généralisé de l'époque; en outre, de nombreuses obscurités ou imprécisions, sinon des affirmations qui ne concordent pas avec la vérité exigeraient une rectification; enfin, on relève, ici et là, des négligences d'écriture qui auraient pu facilement être corrigées. 
M. Jolois consacre tout d'abord une quarantaine de pages, au début de son ouvrage, à la description du "contexte canadien": milieu géographique, déclin économique, l'Eglise et la survivance du Canada français, la société canadienne-française et l'organisation scolaire. Cette pièce de bravoure est-elle destinée à des lecteurs français qui ignorent tout du Canada et avec qui on peut en prendre à son aise avec les assertions historiques qu'ils ne sont pas en état de vérifier, ou est-elle écrite à l'intention des Canadiens d'expression française qui connaissent l'histoire de leur pays? Nous estimons que cette partie de l'ouvrage fait figure de hors-d'œuvre, qu'elle tient plus du folklore et de la fantaisie que des réalités historiques. Voici deux exemples de cette fantaisie :

Entre 1790 et 1837 , lors de la montée du nationalisme laïque, nombreux furent les hommes qui agirent sur les plans politique et culturel afin de sortir le peuple de sa léthargie; cette double action avait pour but de soustraire la population à l'inévitable assimilation qu'entraînait l'asservissement économique et moral (p. 34).

Qu'est-ce que ce nationalisme laïque ? Quels furent ces hommes qui agirent ainsi sur les plans politique et culturel ? Qu'entend l'auteur par cet asservissement moral? Est-ce la soumission aux lois de l'Eglise ? Nous aimerions bien avoir des réponses claires à ces questions. L'auteur écrit plus loin :

Fidèles à la philosophie laïque, les libéraux canadiens-français s'efforcèrent alors d'intégrer l'instruction publique à l'Etat et les maîtres laïques envahirent peu à peu l'enseignement primaire où ils répandirent l'instruction libérale et les principes de l'école neutre prônés par les philosophes (p. 54).

Voilà une pièce d'éloquence qui aurait besoin d'un exégète : qu'est-ce d'abord que cette philosophie laïque ? Quels sont ces libéraux canadiensfrançais dont parle M. Jolois ? Il ne faut pas oublier que nous sommes en 1766 et que, dans le même paragraphe, il cite le témoignage de Mgr Briand. Et puis, je ne vois pas comment ces "libéraux canadiens-français" s'efforceraient d'intégrer l'instruction publique à l'Etat, en 1766 : la première loi scolaire, destinée surtout à la population anglaise, fut votée en 1801; la seconde, qui profita à tout le Bas-Canada, fut adoptée en 1829. Je ne comprends vraiment pas cette manœuvre d'intégration de l'instruction publique à l'Etat. Même les lois de 1829 et de 1832 laissèrent au clergé catholique et protestant un rôle important à jouer dans le domaine de l'enseignement. Et les maîtres laïques qui envahissent l'enseignement primaire ? Il n'y en avait pas d'autres pour diriger les écoles de garçons et ils étaient fort peu nombreux; les Frères des Ecoles chrétiennes n'arriveront à Montréal qu'en 1837 ! Et ces maîtres qui répandirent l'instruction libérale ? que veut dire l'auteur par cette expression? Il s'agit sans doute de "principes de l'école neutre prônés par les philosophes ?" Les pauvres maîtres de l'époque étaient bien incapables de ces subtilités philosophiques... Au demeurant, cette citation illustre bien la manière de l'auteur qui se complaît dans les généralités sans se donner toujours la peine de prouver par des faits ce qu'il avance. 
Le témoignage de l'abbé V.-C. Fournier, curé de La Baie du Febvre, qu'il cite à cette occasion, ne suffit pas à justifier les conclusions très générales qu'en tire l'auteur.

M. Jolois, pour défendre les écoles multiconfessionnelles et neutres de Perrault, manifeste, tout au long de son ouvrage, une aggressivité persistante et même agaçante contre le clergé catholique et l'Eglise catholique romaine. Nous ne pouvons expliquer cette attitude que par l'ignorance de l'œuvre accomplie par l'Eglise catholique sous le régime français d'abord, puis, après la conquête, pour assurer la survivance du fait français en cette terre d'Amérique. A moins que l'auteur ne veuille, au départ, nier l'évidence, admise par tous les historiens impartiaux, même non catholiques ? Voici quelques exemples de cette aggressivité: Perrault

... lutta pour établir une société nouvelle, laïque et technique, qui devait remplacer la société théocratique et agraire du Bas-Canada, en ce début du XIX $\mathrm{XI}^{\ominus}$ siècle (p. 15).

Quelques pages plus loin, l'auteur écrit encore :

Arrêtée brutalement dans son évolution économique, déjà lente et pénible, la société rurale inhibée par les événements se replia douloureusement sur elle-même; elle fut encouragée en cela par l'Eglise canadienne et par l'administration britannique qui, sans chercher les mêmes buts, atteignirent la même fin: l'engourdissement et l'apathie de la communauté française du Saint-Laurent. Le clergé voyait enfin se réaliser le rêve si longtemps caressé sous le régime français ... Mais pour créer ce royaume de Dieu en terre canadienne, il fallait que le peuple s'impose certains sacrifices; ce peuple ainsi prédestiné devait renoncer aux biens temporels afin de spiritualiser son destin (pp. 48-9).

En guise d'appui à ce texte, l'auteur cite un passage de Mgr L.-F. Laflèche qui mourut en 1898 et qui fut intimement mêlé à la querelle de l'ultramontanisme; quelques pages plus loin, il fera appel (sans le nommer) à Mgr André-Albert Blais, deuxième évêque de Rimouski et qui vécut de même à la fin du XIX ${ }^{\mathrm{e}}$ siècle. Cette transposition du climat religieux d'une époque à une autre fausse gravement l'interprétation des faits et la mentalité qui prévalait à l'époque où vivait Perrault; cette distorsion de l'histoire sert peut-être la cause que veut défendre M. Jolois, mais elle dessert celle de la vérité historique.

Et voici d'autres passages de même trempe :

L'autorité de l'Eglise ainsi reconnue, les mandements épiscopaux et les directives des curés conditionnèrent la prise de conscience d'un groupe. Ce n'était pas une simple obéissance des individus au clergé mais plutôt une assimilation presque totale de la société rurale à l'Eglise : cette population vivait, pensait et agissait au rythme de l'Eglise dans toutes les sphères de sa conscience et de son activité (p. 50-1). 
Au Canada, comme en France, l'élite laïque refusa de reconnaître à l'Eglise la pleine et entière responsabilité de l'éducation populaire (p. 53).

La société rurale n'évolua guère au cours de ces années (au début du $\mathrm{XIX}^{\mathrm{e}}$ siècle). Un peu plus repliée sur elle-même, psychologiquement structurée par deux siècles de misère, d'abandon, d'apathie, elle se tourna vers Dieu et vers la terre dont elle attendait tout (p. 59).

En voulant diffuser et implanter en terre canadienne la notion des droits de l'homme, le respect des convictions religieuses, les structures d'un Etat laïque et d'un enseignement neutre, les droits à la liberté de pensée et de parole, les libéraux laïques dressèrent l'Eglise contre leur action (p. 64).

En fait, l'école de Perrault était une réaction normale et saine à la formation essentiellement religieuse qui était dispensée dans la plupart des écoles paroissiales (p. 140).

Nous pouvons aussi supposer, bien que rien ici ne l'atteste, (ce n'est pas très scientifique pour un historien!) que l'Ecole rencontra l'opposition du clergé des paroisses rurales... (p. 164-165).

De son côté, le clergé ne facilita pas le développement des établissements scolaires primaires et il dut, afin de défendre la suprématie de l'Eglise catholique, écarter tout ce qui pouvait paraître néfaste à la santé spirituelle de ses ouailles; aussi, préféra-t-il parfois sacrifier l'instruction à l'intégrité religieuse de la société catholique (p. 167).

Nous pouvons comprendre les raisons qui motivèrent l'opposition d'une partie du clergé canadien-français à l'égard de Perrault. Les événements politiques avaient placé la société du Bas-Canada hors des grands courants économiques et industriels des $\mathrm{XVIII}^{\mathrm{e}}$ et $\mathrm{XIX}^{\mathrm{e}}$ siècles, et par ailleurs, la formation religieuse et morale devait nécessairement primer dans un système scolaire conçu pour établir "le royaume de Dieu sur terre". C'est ainsi que pour s'opposer à l'élément britannicoprotestant, qui fondait ses structures sociales sur le capitalisme industriel, l'on parvint à définir la pauvreté du paysan canadien-français comme un don divin; aussi l'orientation sociale nouvelle préconisée par Perrault et la formation professionnelle jointe à l'instruction élémentaire semblèrent porter atteinte au droit naturel de Dieu (sic)". (p. 195-196) ... N'ayant jamais eu à prendre conscience de la montée des masses prolétariennes et de l'évolution industrielle, le clergé canadien considéra l'ouvrier des villes comme un individu douteux, voire même comme un révolutionnaire dangereux (p. 196). 
Nous laissons au lecteur le soin de juger de la validité et de la véracité des assertions de M. Jolois; nous avons souligné quelques passages qui mériteraient qu'on s'y arrêtât davantage afin d'essayer de comprendre la pensée profonde de leur auteur. Il faudrait sans doute une définition claire et nette de ce qu'il entend par cette élite laïque, dont il parle sans cesse, évitant de donner des noms. On pense évidemment à Perrault lui-même, un peu à Louis-Joseph Papineau; mais qui sont les autres? En outre, l'auteur s'en prend à plusieurs reprises à Mgr Jean-Jacques Lartigue, évêque à Montréal, dont on connaît les "saintes colères" et les attaques contre les écoles de Perrault : mais Montréal était alors bien loin de Québec et ces attaques se bornaient à des considérations épistolaires qui n'eurent que bien peu d'influence sur les écoles québecoises.

Quant aux obscurités, imprécisions, inexactitudes, qu'il suffise d'en donner ici quelques exemples. Parlant de la situation après 1760 , l'auteur écrit :

La misère atroce et généralisée qui s'étendit alors sur la colonie, astreignit le peuple à tenter de reconstruire ce qui avait été détruit (qu'est-ce qui avait été détruit au juste ?) et il ne se préoccupa guère d'analyser le contenu idéologique du nouveau régime (p. 45).

Perrault décide un jour de se présenter comme candidat aux élections du Bas-Canada; mais sentant qu'il ne pourrait être élu, "il se retira le second jour lors de l'ajournement" (p. 109). Que veut dire l'auteur? De quel ajournement s'agit-il ici ?

Le 8 avril 1836, Perrault offre ses services comme professeur en chef à l'Ecole normale qu'on projette d'établir à Québec (il est alors âgé de 83 ans !). M. Jolois déplore qu'on l'ait refusé et affirme que, sous sa direction, l'institution aurait rempli ses fonctions (p. 175). Rappelons que l'Ecole normale de Québec ne fonctionna jamais; seule celle de Montréal dispensa l'enseignement de 1837 à 1842. Quelques pages plus loin, l'auteur parle de la pensée pédagogique et philosophique de Perrault. On peut se demander s'il en eut vraiment une car, à la page 185, l'auteur déclare que "le plagiat" lui permit d'élaborer sa propre pensée pédagogique, qu'il se rendit coupables de répétitions, de contradictions et que son cuvre (?) présente un amalgame confus. M. Jolois écrit même : "Nous devons admettre que cette étude malgré les nombreux textes accessibles ne parvient pas à découvrir ou à définir ce que Perrault apporta réellement aux hommes de son temps!" (p. 187). Peut-être le texte qui suit nous permettra-t-il de pénétrer dans les arcanes de la philosophie de Perrault... et de celle de M. Jolois :

Alors que traditionnellement, écrit ce dernier, l'orientation de l'instruction se faisait selon un axe vertical afin de mener l'enfant à la découverte de la connaissance divine, la conception mésologique d'une relation structurelle horizontale souleva une brutale opposition (p. 249). 
Les années 1820 à 1837 auraient déterminé "l'influence de Perrault sur la création et notamment sur le développement du système scolaire de la province du Bas-Canada". Nous regrettons de ne pouvoir partager l'opinion de $\mathrm{M}$. Jolois à ce sujet : l'organisation du système scolaire durant ces dix-sept années ne fut sûrement pas due à l'influence de Perrault; il n'y a qu'à relire l'histoire des lois scolaires de 1824,1829 et 1832 pour comprendre que les législateurs ne s'inspirèrent pas du philosophe de 1' "Asile champêtre". L'une des plus belles perles de l'ouvrage de M. Jolois, c'est sûrement la déclaration suivante qui figure dans l'avant-propos :

Et son destin fut dramatique; incompris des hommes, il (Perrault) connut le sort de Galilée, de Rousseau, de Lamennais, de Teilhard de Chardin et de tant d'autres encore qui ont été désavoués pour n'avoir pas épousé servilement les idées de leur époque (p. 15).

Comparer Joseph-François Perrault à Galilée, à Teihard de Chardin, c'est un comble ! Et voici une autre découverte : parlant des conditions géographiques du Canada, l'auteur écrit sérieusement :

La NEIGE fut certainement un des principaux facteurs de l'organisation des structures familiales et sociales du Canada français (p. 39).

Et il ajoute quelques lignes plus loin :

Et le village ... perdit sa raison d'être ... Le village n'existant pas (???) ou se réduisant souvent à un clocher, la centralisation et le développement de la vie administrative, judiciaire, politique et culturelle ne purent se faire normalement (p. 40).

Un village qui se réduit à un clocher, c'est tout un village! Rappelons à l'auteur qu'en 1756 , il y avait 88 paroisses régulièrement organisées, plus 16 desservies par un missionnaire, plus 19 autres endroits où l'on demandait des églises, affirme le chanoine J.-O. Briand, preuve évidente que dans ces agglomérations, il y avait des habitants groupés autour du clocher !

Autre inexactitude: Les conditions économiques "qui furent l'apanage de la société du Bas-Canada au cours de la première moitié du XIX ${ }^{\mathrm{e}}$ siècle entravèrent l'organisation de tout système scolaire" ( $p$. 48). Que fait l'auteur des lois scolaires de 1801, 1824, 1829, 1832, 1836, 1841, 1845, 1846 ? C'est vraiment trop simple d'imputer aux conditions économiques de l'heure les lacunes de l'organisation scolaire, car il y eut une organisation scolaire, grâce à la loi de 1801 , grâce surtout à celles de 1829 et de 1832 , pour ne parler que de la période avant 1837.

Nous ne pouvons non plus accepter l'assertion suivante : "Il semble (ce n'est donc pas sûr ?) que, de 1760 à 1841, le clergé se préoccupa assez peu de l'enseignement primaire" (p. 55). L'auteur oublie-t-il les démarches qui firent adopter la Loi des Ecoles de Fabriques en 1824 et la collaboration loyale que le clergé apporta à la mise en œuvre des lois scolaires de 1829 et 1832 (loi des Ecoles de Syndics), l'insistance des évêques auprès des curés pour l'établissement d'écoles élémentaires? 
Les événements politiques se précipitent sous la plume de $\mathrm{M}$. Jolois qui écrit (p. 149): "L'agitation politique, commencée dès 1792 (à prouver), dégénéra rapidement en insurrection après 1836 !". Ce fut sans doute une mini-insurrection qui prit 45 ans pour arriver à maturité; comme vitesse d'accélération, ce ne fut donc pas très rapide.

A la page 192, l'auteur affirme que "l'orientation des écoles canadiennes était nettement définie en 1820": nous aimerions bien savoir par qui, par quoi, cette orientation fut faite. Par la loi scolaire de 1801, la seule jusqu'alors, et qui fut à peu près inutilisée par les Canadiens français ? Mystère.

Parlant de la période de 1801, M. Jolois écrit : “A ce moment, ni les nationalistes laïques ni le clergé ne réagirent" (p. 247). Encore une fois, nous aimerions connaître les noms de ces nationalistes laïques en 1801! (Louis-Joseph Papineau n'avait alors que 16 ans!)

Enfin, relevons aux pages 250 et 251 , deux déclarations péremptoires : l'œuvre de Perrault "fut assurément la plus considérable réalisée au XIX siècle" et "il paraît impossible de nier qu'avant Perrault l'éducation élémentaire n'existait pas". Nous regrettons de devoir nous inscrire en faux contre de telles affirmations qui ne sont pas conformes à la vérité : Perrault ne fut pas le seul pédagogue du XIX $\mathrm{X}^{\mathbf{e}}$ siècle au Canada français. Il suffit de mentionner les abbés Jérôme Demers, John Holmes, Jean Langevin, H.-A. Verrault, MM. Urgel-Eugène Archambault, Jean-Baptiste Meilleur, P.-J.-O. Chauveau et nombre d'autres, sans parler de ces pédagogues modestes des communautés religieuses enseignantes. Bien plus, l'éducation élémentaire exista avant Perrault : les couvents des Sœurs de la Congrégation de NotreDame, des Ursulines, des Sœurs de l'Hôpital général de Québec et ces très nombreuses écoles qui surgirent en vertu des lois de 1801, 1824, 1829 et 1832 exercèrent, à ce palier, une influence qu'il serait injuste de sous-estimer.

Nous nous excusons de cet "échenillage" laborieux : il nous a semblé indispensable cependant, à la suite des recensions qui sont parues de cet ouvrage et qui toutes, même celle de Jean-Ethier Blais, dans le Devoir du 25 octobre 1969, n'ont que des éloges à formuler à l'endroit de l'auteur et de son héros. Pour notre part, nous estimons que Joseph-François Perrault ne méritait pas tant d'efforts, ni un piédestal de cette qualité. Paradoxe assez singulier, M. Jolois admet lui-même que Perrault "ne fut pas génial, qu'il fut mesquin, cupide, médiocre", alors que la plus grande partie de son livre tend à démontrer le contraire et à nous le faire admirer comme le pionnier de l'enseignement laïque au Bas-Canada; bref, ce pauvre Perrault a eu raison contre tous ! C'est pourquoi, souligne M. Jolois, son œuvre a sombré dans l'oubli ! A notre avis, elle devrait y rester... en attendant la résurrection des morts.

Louis-Philippe Audet, de la Société royale du Canada

Faculté des Sciences de l'Education

Université de Montréal, Montréal 